\section{Expression of immediate-early genes in the inferior colliculus and auditory cortex in salicylate-induced tinnitus in rat}

\author{
S.S. Hu, ${ }^{1,2}$ L. Mei, ${ }^{1,2}$ J.Y. Chen, ${ }^{1,2}$ \\ Z.W. Huang, ${ }^{1,2}$ H. $W_{u^{1,2}}$ \\ 'Department of Otolaryngology-Head \\ and Neck Surgery, Xinhua Hospital, \\ Shanghai Jiao Tong University School \\ of Medicine, Shanghai \\ 'Ear Institute, Shanghai Jiao Tong \\ University School of Medicine, Shanghai, \\ China
}

\begin{abstract}
Tinnitus could be associated with neuronal hyperactivity in the auditory center. As a neuronal activity marker, immediate-early gene (IEG) expression is considered part of a general neuronal response to natural stimuli. Some IEGs, especially the activity-dependent cytoskeletal protein (Arc) and the early growth response gene-1 (Egr-1), appear to be highly correlated with sensory-evoked neuronal activity. We hypothesize, therefore, an increase of Arc and Egr-1 will be observed in a tinnitus model. In our study, we used the gap prepulse inhibition of acoustic startle (GPIAS) paradigm to confirm that salicylate induces tinnitus-like behavior in rats. However, expression of the Arc gene and $E g r$ - 1 gene were decreased in the inferior colliculus (IC) and auditory cortex (AC), in contradiction of our hypothesis. Expression of $N$-methyl D-aspartate receptor subunit 2B (NR2B) was increased and all of these changes returned to normal 14 days after treatment with salicylate ceased. These data revealed long-time administration of salicylate induced tinnitus markedly but reversibly and caused neural plasticity changes in the IC and the AC. Decreased expression of Arc and Egr-1 might be involved with instability of synaptic plasticity in tinnitus.
\end{abstract}

\section{Introduction}

Tinnitus is the perception of sound in the absence of an external acoustic stimulus. Prolonged tinnitus frequently has a marked negative impact on the quality of life, causing a great deal of psychological distress. ${ }^{1}$ Owing to the increasing use of personal headsets, especially among young adults, ${ }^{2}$ tinnitus presents a serious health issue. The exact mechanism underlying tinnitus is unclear. Animal models have contributed to understanding the mechanism underlying tinnitus, and salicylate-induced tinnitus in rats serves as a popular animal model for the study of tinnitus. ${ }^{3-5}$ Earlier studies showed long-term administration of salicylate can increase the distortion product of acoustic emissions, ${ }^{6}$ increase outer hair cell electromotility, ${ }^{5,7}$ modify the average spectrum of electrophysiological cochleoneural activity ${ }^{8,9}$ and induce tinnitus reliably.

Several pieces of evidence indicate spontaneous neuronal activity in auditory regions might be involved in the physiological processes underlying salicylate-induced tinnitus. An increase to various degrees in the spontaneous activity of auditory neurons after administration of salicylate has been reported at the level of the auditory nerve,,$^{8,9}$ the inferior colliculus (IC) $)^{10,11}$ and the auditory cortex (AC). ${ }^{12,13}$ Salicylate can have a direct influence on the spontaneous activity of different types of neurons in the central auditory system in an in vitro brain slice. ${ }^{14,15}$ Immediate-early gene (IEG) activation is considered part of a general neuronal response to natural stimuli and a result of normal synaptic activity. The immunohistochemistry of IEG expression is a useful method to visualize activated neuronal populations in the brain of animals. ${ }^{16}$ IEG expression reflects recent neuronal activity. Some IEGs, especially Arc or Egr-1, appear to be highly correlated with sensory evoked neuronal activities, ${ }^{17}$ and they are induced rapidly in neurons by patterned synaptic activity that activates $N$-methyl-D-aspartate (NMDA) receptors. ${ }^{18}$ However, there are few reports of their relationship with tinnitus induced by longtime administration of salicylate.

In this study, the gap prepulse inhibition of acoustic startle (GPIAS) paradigm was used to detect salicylate-induced tinnitus-like behavior in rats. ${ }^{19-21}$ We investigated the expression levels of Arc, Egr-1 and NMDA receptor subunit $2 B$ (NR2B) genes in the IC and $\mathrm{AC}$ in response to acute and chronic administration of salicylate. Interestingly, $A r C$ and Egr-1 expression was reduced significantly in the IC and AC. Reduction of both Arc and Egr-1 expression might be involved with instability of homeostatic plasticity in tinnitus.

\section{Materials and Methods}

\section{Animals}

Experimental procedures were approved by the Animal Care and Use Committee of the Shanghai Jiao Tong University School of Medicine. A total of 48 adult male Sprague Dawley $^{\circledR}$ rats (body weight 250-350g) were divided into four groups on the basis of our earlier study:6,7 i) control group $(n=9)$; ii)
Correspondence: Prof. Zhi-Wu Huang, Department of Otolaryngology-Head \& Neck Surgery, Xinhua Hospital, Shanghai Jiao Tong University School of Medicine, 1665 Kongjiang Road, Shanghai 200092, China.

Tel. +86.21.25078892 - Fax:+86.21.65156489.

E-mail: huangzw86@gmail.com

Key words: salicylate, inferior colliculus, auditory cortex, activity-dependent cytoskeletal protein, early growth response gene-1.

Contribution: SSH, laboratory experiments, manuscript drafting; ZWH, study concept and design; LM, JYC, statistical analysis performing; HW, study supervision. All authors participated in the revision of the manuscript and have given final approval for the manuscript to be published.

Conflict of interests: all authors declare no conflict of interests.

Received for publication: 3 September 2013. Accepted for publication: 31 January 2014.

This work is licensed under a Creative Commons Attribution NonCommercial 3.0 License (CC BYNC 3.0).

○Copyright S.S. Hu et al., 2014

Licensee PAGEPress, Italy

European Journal of Histochemistry 2014; 58:2294 doi:10.4081/ejh.2014.2294

acute treatment group with salicylate injected once $(n=6)$; iii) chronic treatment groups with daily injections of salicylate for 3 days $(n=6)$ (S3), 7 days $(n=6)(\mathrm{S} 7)$ or 14 days $(n=9)$ (S14); iv) recovery groups with 14 days $(n=6)$ (S14+R14) and 28 days $(\mathrm{n}=6)(\mathrm{S} 14+\mathrm{R} 28)$ post-chronic salicylate administration.

\section{Design and salicylate administration}

Sodium salicylate (Sigma-Aldrich, Shanghai, China) was dissolved in normal saline ( $9 \%$ $(\mathrm{w} / \mathrm{v}) \mathrm{NaCl}$ ) at a final concentration of 200 $\mathrm{mg} / \mathrm{mL}$. Rats in the acute treatment group received a single intraperitoneal injection of salicylate $(400 \mathrm{mg} / \mathrm{kg})$. Rats were anesthetized deeply with sodium pentobarbital $(40 \mathrm{mg} / \mathrm{kg}$, administered intraperitoneally) and sacrificed $2 \mathrm{~h}$ later. Rats in the chronic treatment groups were given an intraperitoneal injections of salicylate $(200 \mathrm{mg} / \mathrm{kg})$ daily at $08: 00 \mathrm{~h}$ and at $16: 00$ h for 3 (S3), 7 (S7) or 14 (S14) consecutive days and were sacrificed at $08: 00 \mathrm{~h}$ on days 4 (S3), 8 (S7) or 15 (S14), respectively. The recovery groups were given intraperitoneal injections for 14 consecutive days and recovery of 14 days (S14+R14) and 28 days (S14+R28), respectively, after cessation of treatment. The control group was given intraperitoneal injections of saline $(200 \mathrm{mg} / \mathrm{kg})$ twice daily at 8:00 and 16:00 for 14 consecutive days. 


\section{Gap detection testing}

Tinnitus was assessed using the GPIAS paradigm as described in detail elsewhere. ${ }^{19,20}$ This program exploits the acoustic startle reflex in animals treated with salicylate. GPIAS testing began $1 \mathrm{~h}$ before rats were sacrificed. Each animal was placed in a permeable sound box resting on a sensitive piezoelectric transducer capable of generating a voltage proportional to the magnitude of the startle response evoked by sound stimuli generated digitally by a digital signal processor (RZ6, Tucker Davis Technologies, Alachua, FL, USA). The amplitude of the startle response was collected by a computer and analyzed offline.

GPIAS sessions consisted of 30 gap trials and 30 no-gap trials. Rats underwent testing with different band-pass-filtered $(1000-\mathrm{Hz}$ bandwidth) sounds centered at 6,12 and 16 $\mathrm{kHz}$ at a sound level of $65 \mathrm{~dB}$ SPL. Startle responses were elicited by a 20 -ms burst of white noise at $100 \mathrm{~dB}$ SPL. The gap in the narrowband noise began $100 \mathrm{~ms}$ before the onset of the broadband startling noise and the last 50 ms. The interval between each startling noise was 30-35 $\mathrm{s}^{20}$ and each test lasted $30 \mathrm{~min}$.

Percentage GPIAS was calculated by computing the average ratio of trials with a gap versus no-gap trials for each frequency using the formula:

\section{[(AvgTnogap - AvgTgap) / AvgTnogap]×100\%}

where AvgTgap was the average amplitude during gap trials and AvgTnogap was the average amplitude of no-gap trials. ${ }^{21,22}$

\section{Quantitative real-time PCR}

Rats were sacrificed after deep anesthesia was induced by an injection of pentobarbital (40 mg/kg body weight). IC and AC were dissected rapidly and total RNA was extracted from each sample with TRIzol reagent according to the manufacturer's protocol. Extracted RNA was quantified spectrophotometrically at 260 and $280 \mathrm{~nm}$. High-quality RNA was reverse transcribed into complementary DNA (cDNA) using a Reverse Transcription Kit (DRR036A, TaKaRa). Primers for Arc, Egr-1, NR2B and glyceraldehyde-3-phosphate dehydrogenase (GAPDH), designed by Takara (Otsu, Japan), were obtained from Shanghai Sangon Biological Engineering Technology \& Services Co., Ltd. The PCR primer sequences were:

Arc-F, 5'-CTGCCACAGAAGCAGGGTGA-3' Arc-R, 5'-AGGGTGCCCACCACATACTGA-3' Egr-1-F, 5'-GAACAACCCTACGAGCACCTG-3' Egr-1-R, 5'-GCCACAAAGTGTTGCCACTG-3' NR2B-F, 5'-TGGCTATCCTGCAGCTGTTTG-3' NR2B-R,5'-TGGCTGCTCATCACCTCATTC-3' GAPDH-F, 5'-GGCACAGTCAAGGCTGAGAATG-3' GAPDH-R,5'-ATGGTGGTGAAGACGCCAGTA-3'
PCR amplification was done with SYBR ${ }^{\circledR}$ Premix Ex Taq ${ }^{\mathrm{TM}}$ (DRR420A, TaKaRa). The PCR protocol was: $95^{\circ} \mathrm{C}$ for $30 \mathrm{~s}$, followed by 40 cycles of $95^{\circ} \mathrm{C}$ for $5 \mathrm{~s}, 60^{\circ} \mathrm{C}$ for $34 \mathrm{~s}$ and a final dissociation stage, using the ABI 7500 realtime PCR system (Applied Biosystems, Foster City, CA, USA). We assumed the amplification efficiency of the target and reference were approximately equal. Relative quantification and calculations were done with the comparative threshold $\left(C_{\mathrm{t}}\right)$ cycle method $\left(2^{-\Delta \Delta C \mathrm{t}}\right){ }^{23}$

\section{Western blot}

Total proteins were extracted from the samples and their concentration was determined using an ultraviolet spectrophotometer (DR/4000UV-VIS, Hach Co., Loveland, C0, USA). SDS-PAGE (12\% (w/v) polyacrylamide gel) was used to electrophorese Arc and Egr-1 protein and SDS-PAGE (8\% (w/v) polyacrylamide gel) was used to electrophorese NR2B protein: equal quantities of protein were loaded. After separation, proteins were transferred electrophoretically to polyvinylidene difluoride membranes, which were blocked in Tris-buffered saline, $0.1 \%(\mathrm{v} / \mathrm{v})$ Tween 20, 5\% (w/v) skimmed milk powder and then incubated with primary antibodies overnight and washed in Tris-buffered saline, $0.1 \%$ Tween 20 . Secondary antibodies were diluted in blocking buffer and incubated with the membranes for $2 \mathrm{~h}$ at room temperature. Finally, the immunoreactive bands were visualized by the SuperSignal Chemilumnescent Substrate system (Pierce). The images of western blot analysis were quantified by Image Lab software, and band intensities of Arc, Egr-1 and NR2B were expressed relative to GAPDH. The following antibodies were used: 1:1000 rabbit polyclonal anti-Arc antibody (ab23382, Abcam, Shanghai, China), 1:1000 rabbit anti-Egr-1 antibody (4153S, Cell Signaling Technology, Danvers, MA, USA), 1:1000 rabbit antiNMDAR2Bantibody (4212S, Cell Signaling Technology) and 1:5000 goat anti-rabbit IgGHRP (Jackson).

\section{Immunohistochemistry}

Rats were anesthetized deeply and perfused through the ascending aorta with normal saline followed by $4 \%$ (v/v) paraformaldehyde. The brain was removed and IC and AC were dissected. Immunostaining was done on paraffin-embedded sections of rat IC and AC, which were then deparaffinized in xylene and rehydrated rapidly through a graded series of alcohol. Excess liquid was removed and the sections were washed in phosphate-buffered saline (PBS), pH 7.4, 0.05\% (v/v) Tween20 (PBS-T). In order to reduce nonspecific binding, normal goat serum (1\% (v/v) in PBS) was applied to slides for $30 \mathrm{~min}$ at $37^{\circ} \mathrm{C}$. The sections were then incubated with primary antibody on consecutive sections. Sections were rinsed with PBS-T then incubated with secondary antibodies for $1 \mathrm{~h}$ at room temperature. The immunoreactions were visualized using $0.015 \%(\mathrm{v} / \mathrm{v}) \mathrm{H}_{2} \mathrm{O}_{2}$ in 3,3'-diaminobenzidinetetrahydrochloride (DAB)/Tris-buffered saline for $10 \mathrm{~min}$ at room temperature. The following antibodies were used: 1:50 rabbit anti-Arc antibody (ab23382, Abcam), 1:50 rabbit anti-Egr-1 antibody (4153S, Cell Signaling Technology) and 1:50 rabbit anti-NMDAR2B antibody (4212S, Cell Signaling Technology).

Immunostaining was assessed quantitatively with the Microimage Analysis Program (Optimas 6.5, Media Cybernetics, Rockville, MD, USA). In brief, sections were placed under a microscope (Olympus BX 50) and the image was transferred to a computer via a digital camera (Nikon CoolPix 950). In each section,

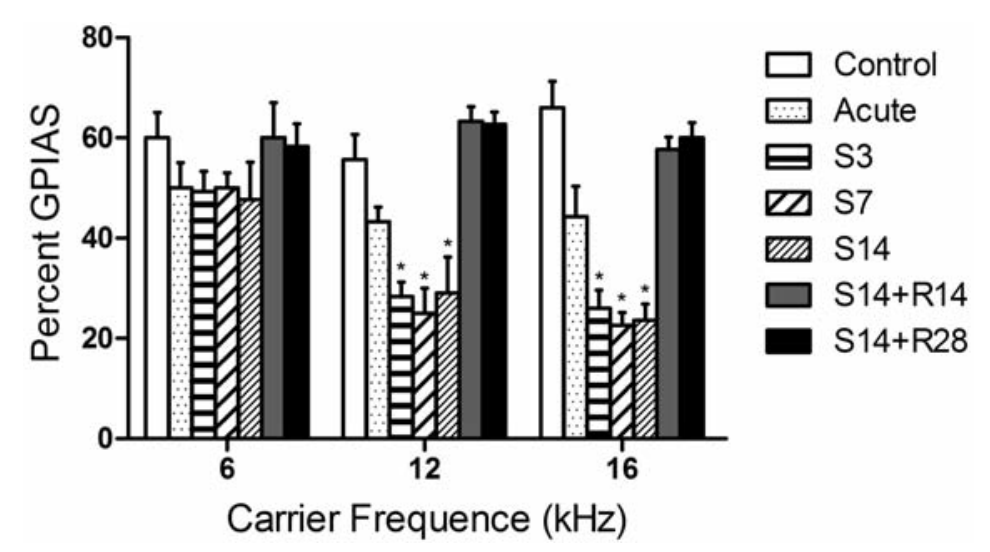

Figure 1. Effects of salicylate on gap prepulse inhibition of acoustic startle (GPIAS) values. Chronic treatment groups (S3, S7 and S14) showed a significant decrease in GPIAS values compared to the control group at $12 \mathrm{kHz}$ and $16 \mathrm{kHz}$ but not at $6 \mathrm{kHz}$. There was no difference of GPIAS values among the acute treatment recovery (S14+R14, S14+R28) and control groups. 
areas of positive immunostaining of neurons in the bilateral hemispheres were selected and the integrated optical density (OD) was measured in three sections from IC and AC in each rat for Arc, Egr-1 and NR2B. The results were expressed as the percentage of relative OD units compared to the control groups, which were given a value of $100 \%$. The OD values obtained in three sections per rat were averaged and used to calculate the group means. Photomicrographs for coronal sections of the IC and AC were taken and analyzed using image $\mathbf{J}$ software to evaluate the number of immunoreactive neurons (IRN). The density of IRN was expressed as mean \pm standard deviation (SD) of the number of positive neurons/section, and the significance of any difference in density of IRN between control and S14 groups for structures on the same side were determined using a two-tailed $t$-test.

\section{Statistical analysis}

All data were calculated and presented as mean \pm standard deviation. According to the distribution of the data and the homogeneity of variance, unpaired, two-sided Student's $t$-test and one-way analysis of variance (ANOVA) followed by Student-Newman-Keuls (SNK) posthoc tests were used for comparisons among groups. The level of statistically significant difference was set at $\mathrm{P} \leq 0.05$.

\section{Results}

\section{Salicylate-induced tinnitus-like behavior in rats}

Chronic treatment groups (S3, S7 and S14) showed a statistically significant decrease in GPIAS values relative to the control group at 12 $\mathrm{kHz}$ and $16 \mathrm{kHz}$ but not at $6 \mathrm{kHz}$, indicating these animals were experiencing tinnitus. However, there was no difference in GPIAS values in any other group, indicating tinnitus-like behavior disappeared 14 days after treatment with salicylate ceased (Figure 1).

\section{Expression of Arc, Egr-1 and NR2B in the inferior colliculus}

Compared to the control group, Arc mRNA expression was down-regulated significantly in the IC in the acute treatment and chronic treatment groups (S3, S7 and S14) and expression of the Arc protein was decreased significantly in the IC in the chronic treatment groups (S3, S7 and S14), but there was no significant change in any other group (Figure 2). The immunoreactivity of Arc in the IC of the chronically treated group (S14) was significantly lower compared to the control group (Figure 3 A,B,C). Egr-1 mRNA expression was decreased significantly in the IC in the acute and chronic treatment groups (S3, S7 and S14) compared to the control group. Expression of the Egr-1 protein was decreased significantly in the IC in the chronic treatment groups (S7 and S14). There was no significant change in any other group (Figure 2). The immunoreactivity of Egr-1 of the salicylate-treated group (S14) was significantly lower in the IC com- pared to the control group (Figure 3A,B,C). NR2B mRNA and protein expression were upregulated significantly in the IC in rats that were chronically administered salicylate (S3, S7 and S14) compared to the control group. There was no significant change in the acute treatment group or recovery groups after treatment with salicylate ceased (Figure 2). The

A

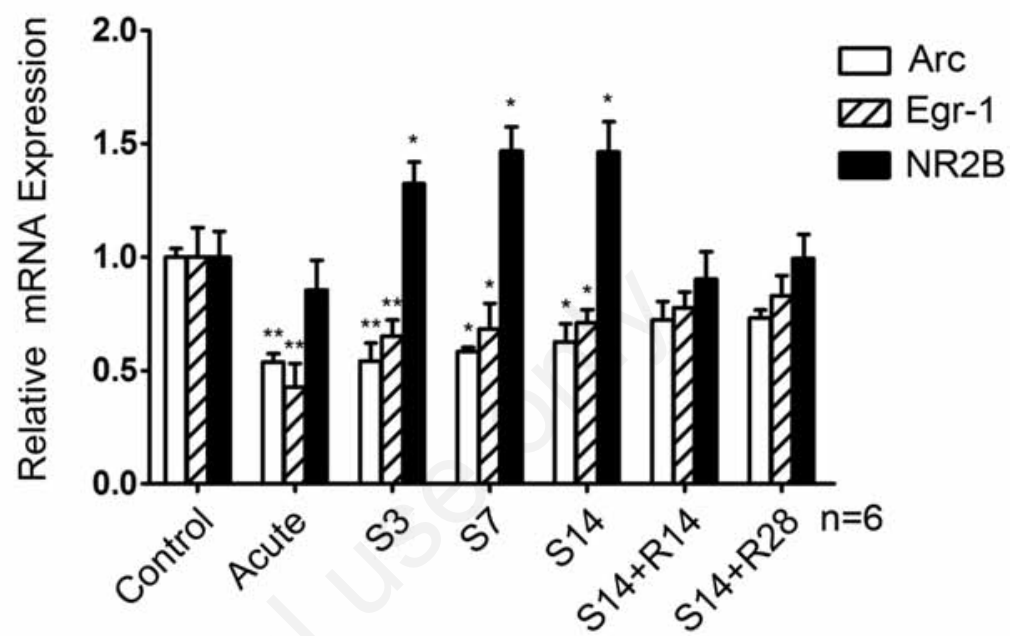

B
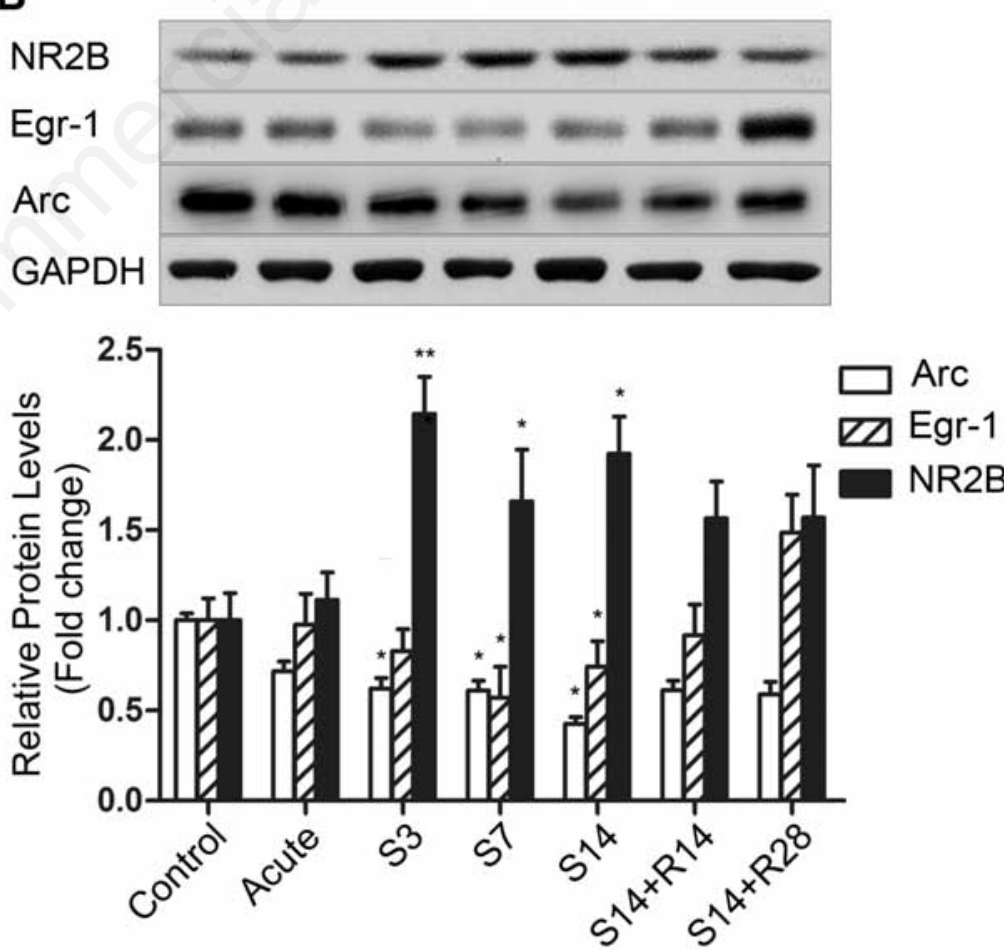

Figure 2. Expression of Arc, Egr-1 and NR2B in the IC. The expression of Arc and Egr1 mRNA was significantly lower in the acute treatment group and chronic treatment groups (S3, S7and S14) compared to the control and recovery groups (S14+R14, $\mathrm{S} 14+\mathrm{R} 28)$. The protein expression of Arc and Egr-1 were significantly lower in the chronic treatment groups (S7 and S14) compared to any other group. However, the expression levels of NR2B (A) mRNA and (B) protein were significantly higher in chronic treatment groups $(\mathrm{S} 3, \mathrm{~S} 7$ and $\mathrm{S} 14)$ compared to any other group. ${ }^{*} \mathrm{P}<0.05$ vs the control group; ${ }^{* *} \mathbf{P}<0.01$ vs the control group. 
immunoreactivity of NR2B in the IC of the chronic treatment group (S14) was significantly higher compared to the control group (Figure $3 \mathrm{~A}, \mathrm{~B}, \mathrm{C}$ ).

\section{Expression of Arc, Egr-1 and NR2B in the auditory cortex}

Compared to the control group, Arc mRNA and protein expression were down-regulated significantly in the AC chronically administered salicylate (S3, S7 and S14). There was no significant change in the acute treatment or recovery groups after treatment with salicylate ceased (Figure 4). The immunoreactivity of Arc in the AC of the chronic treatment group (S14) was significantly lower compared to the control group (Figure 5A,B,C). Expression of Egr-1 mRNA was decreased significantly in the $\mathrm{AC}$ in the chronic treatment groups (S3, S7 and S14) and expression of the Egr-1 protein was decreased significantly in the AC in the chronic treatment groups (S7 and S14) compared to the control group. There was no significant change in the acute treatment group or recovery groups after treatment with salicylate ceased (Figure 4). The immunoreactivity of Egr-1 of the salicylate-treated group (S14) was significantly lower in the $\mathrm{AC}$ compared to the control group (Figure 5A,B,C). The relative mRNA and protein expression levels of NR2B under different salicylate treatment regimens. The levels of mRNA and protein expression of NR2B were up-regulated significantly in the $\mathrm{AC}$ in rat groups chronically administered salicylate (S3, S7 and S14) compared to the control group. Moreover, the increase was not found in the acute treatment group nor was it observed in the recovery groups (S14+R14, S14+R28) after treatment with salicylate ceased (Figure 4). The immunoreactivity of NR2B in the AC of the chronic treatment group (S14) was significantly higher compared to the control group (Figure 5A,B,C).

\section{Discussion}

The expression of IEGs (ArC and Egr-1) is clearly attuned to altered neural activity and synaptic efficacy in neurons as well as related plasticity changes, and they have been validated as an indirect marker of neuronal activity in the hippocampus, ${ }^{24}$ amygdala ${ }^{25}$ and sensory cortices. ${ }^{26-29}$ Hyperactivity in the auditory center is considered to be associated with tinnitus, and earlier reports believed that salicylateinduced tinnitus was associated with upexpression of Egr-1 and NR2B genes in the cochlear. ${ }^{5,30}$ We hypothesize, therefore, an increase of Arc and Egr-1 will be observed in auditory center in the tinnitus model. In this study, however, we found expression of both
$A r C$ and Egr-1 genes was decreased significantly in the IC and $\mathrm{AC}$ in rats with tinnitus, whereas expression of $N R 2 B$ was increased following long-term administration of salicylate. All of these changes had returned to normal 14 days after treatment with salicylate ceased.

Earlier studies showed ${ }^{5,30}$ the NMDA receptor has the major role in inducing Arc or Egr-1 expression. ${ }^{18,31-33}$ and some NMDA receptor antagonists, such as Gacyclidine, ${ }^{34}$ might be potent drugs for the suppression of sen- sorineural tinnitus in humans and, therefore, should be considered for long-term human use and warrant clinical trials. However, expression of Arc or Egr-1 was inconsistent with increased expression of NR2B in our study. NMDA receptor activation is not the sole mechanism with which to induce Arc mRNA transcription and Arc transcription is under the control of both NMDA and $\alpha$-amino-3hydroxy-5-methyl-4-isoxazolepropionic acid (AMPA) receptors. ${ }^{35}$ The cytoskeletal protein
A

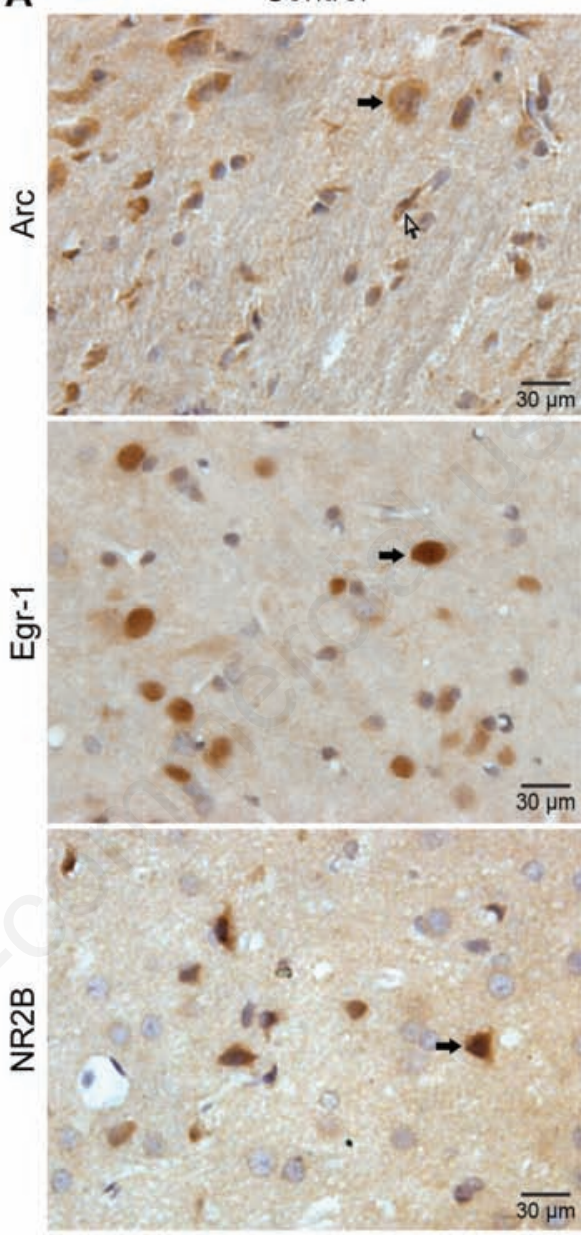

B

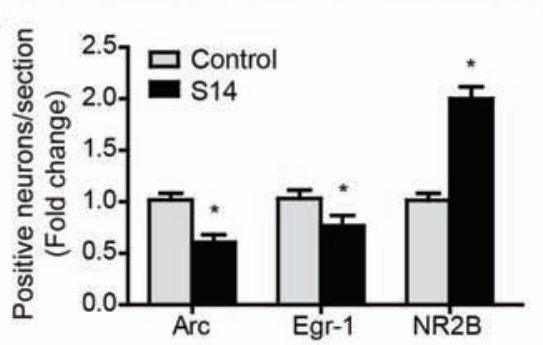

$\mathrm{S} 14$
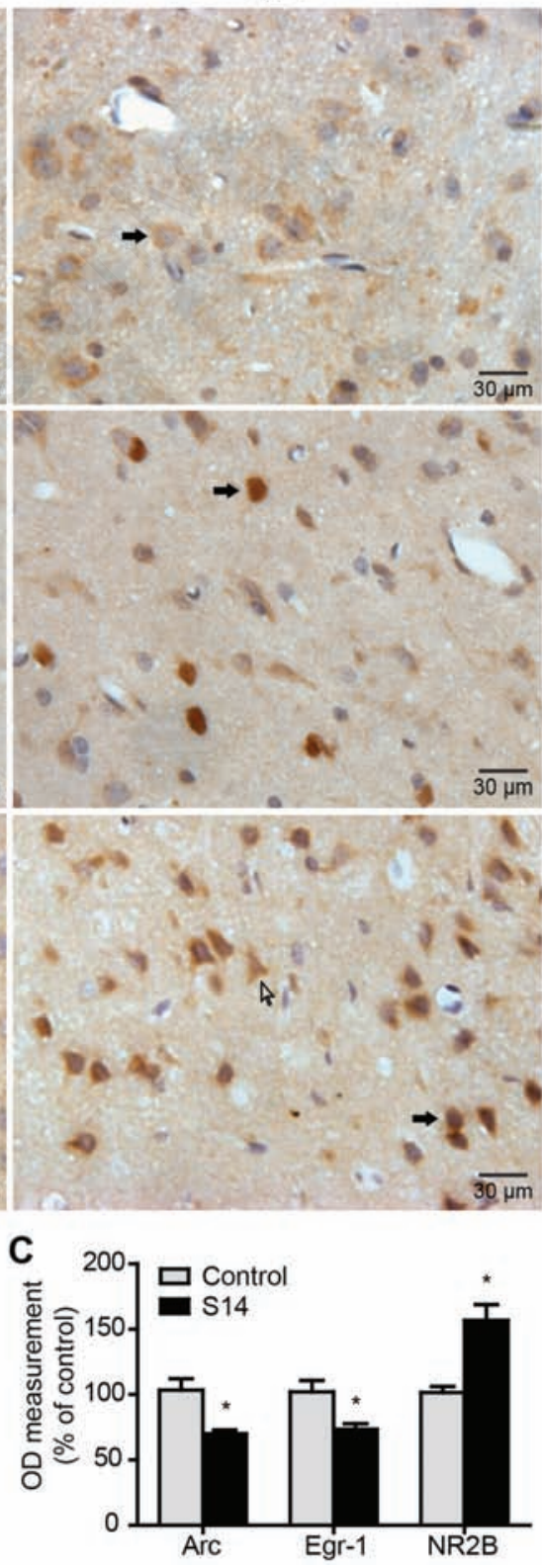

Figure 3. The expression of Arc, Egr-1 and NR2B was evaluated using immunohistochemistry staining (magnification $400 \times$ ) in the IC. A) Immunohistochemical staining of Arc, Egr-1 and NR2B in the IC. Black arrows indicate neurons and white arrows indicate glia. B) The number of Arc, Egr-1 and NR2B positively stained cells in IC. C) Bars show percentage difference in OD compared to the control group $(100 \%)$. Values are given as mean $\pm \mathrm{SD}, \mathrm{n}=6 .{ }^{*} \mathrm{P}<\mathbf{0 . 0 5} v$ s the control group. Scale bars: $30 \mu \mathrm{m}$. 
Arc is increased following long-term potentiation (LTP)-like activity of sustained scale AMPA receptors in postsynaptic membranes of dendritic spines, ${ }^{36}$ which increases sensitivity to glutamate and synaptic strength. ${ }^{37}$ Moreover, Arc-mediated synaptic scaling is essential for neurons in response to continuous activity changes, maintaining averaged firing rate ${ }^{38}$ and homeostatic adaptation.

Sometimes, unrestrained neuronal input can result in saturation of the neuronal ability to encode information. ${ }^{39}$ Homeostatic compensation for these alterations in synaptic strength is required to maintain neuronal output in the normal range. For example, chronic blockade of network activity for several days resulted in an increase in surface and synaptic AMPA receptors, whereas a chronic increase in activity reduces the number of surface and synaptic AMPA receptors. ${ }^{40}$ Arc is essential for mediating the homeostatic scaling of AMPA receptors. Over-expression of Arc blocks the up-regulation of surface AMPA receptors and miniature excitatory postsynaptic currents (mEPSCs) induced by chronic neuronal inactivity. Conversely, Arc knockout (K0) neurons exhibit a scaled increase in surface AMPA receptors and AMPA receptor-mediated mEPSCs, which mimics the up-regulation of synaptic function induced by chronic inactivity. Arc protein is regulated dynamically by chronic changes in neuronal activity that normally evokes synaptic scaling ${ }^{36}$ and is highly sensitive to plasticity, specifically homeostatic scaling. ${ }^{41}$ Decreased Arc expression would result in abnormal synaptic scaling and instability of long-lasting forms of synaptic efficacy and synaptic plasticity in rats with tinnitus, which appeared to have maladaptive neuroplastic brain alterations. Arc expression returned to normal after 14 days accompanied by the disappearance of tinnitus and restoration of normal synaptic plasticity.

Reduction of Arc, as shown here for animals with tinnitus, results in loss of the normal scaling responses to changes of neuronal activity. ${ }^{36}$ Hippocampal neurons derived from Arc/Arg3.1 KOs displayed larger basic mEPSCs and failed to undergo synaptic scaling adequately. ${ }^{36}$ Moreover, the highly synchronized epilepticlike cortical network activity could be observed in the cortex following Arc decline. ${ }^{42}$ The abnormally high degree of synchronization and epileptic-like neuronal activity in $\mathrm{AC}$ are assumed to be associated with tinnitus. ${ }^{43}$ By application of different models of tinnitus, the decline of Arc mRNA was observed following a strong acoustic trauma induced with a sound of $120 \mathrm{~dB}$ SPL for $2 \mathrm{~h}^{41,44}$ The failure to mobilize Arc in the cortex could suggest that tinnitus is linked to a failure to adapt central circuits to reduced cochlear input.

Egr-1 is expressed in the neocortex, primary olfactory cortex, entorhinal cortex, hippocampus, amygdaloid nuclei, nucleus accumbens, striatum and cerebellar cortex of rat brain. ${ }^{45}$ Egr-1 shows some characteristics similar to those of Arc; they share some of the binding sites on the promoter region, and both are activated by the mitogen-activated protein (MAP) kinase pathway. ${ }^{46,47}$ Moreover, Egr-1 is a molecular marker of sensory input ${ }^{48}$ and has a crucial role in maintenance of late-phase LTP $^{49}$

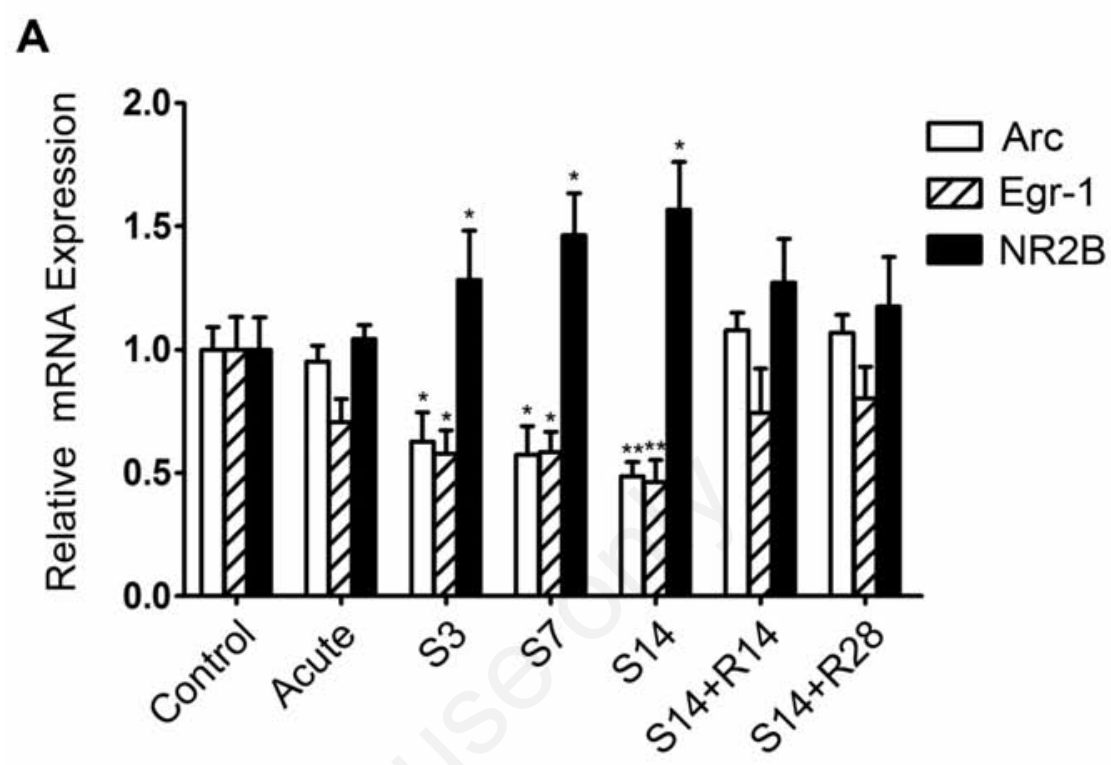

B
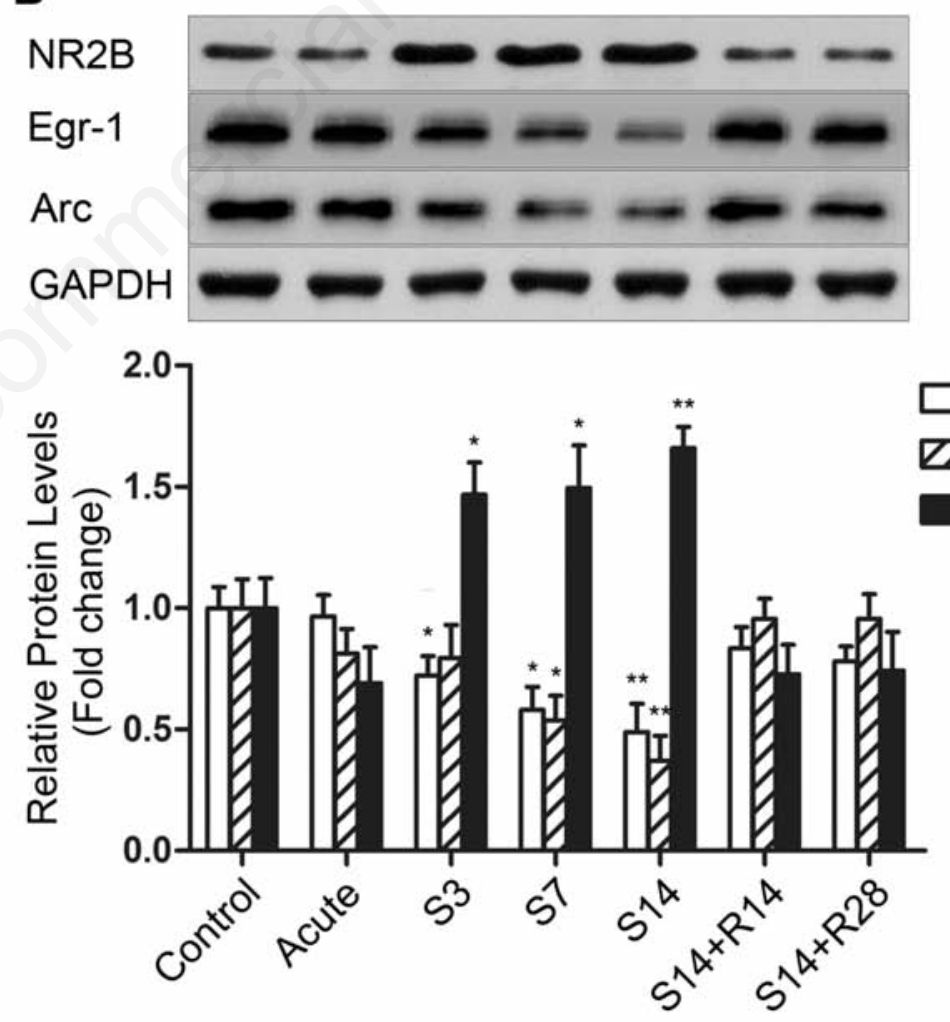

Figure 4. Expression of Arc, Egr-1 and NR2B in the AC. The expression levels of Arc and Egr-1were significantly lower in rats chronically administered with salicylate (S3, S7 and S14) compared to the control, acute treatment and recovery groups (S14+R14, S14+R28), indicated by real-time PCR and western blot assays. However, the expression levels of NR2B (A) mRNA and (B) protein were significantly higher in chronic treatment groups $\left(\mathrm{S} 3, \mathrm{~S} 7\right.$ and S14) compared to any other group. ${ }^{*} \mathrm{P}<0.05$ vs the control group; ${ }^{* *} \mathrm{P}<0.01$ vs the control group. 
Egr-1 deletion has been shown to lead to impaired in vivo late-phase LTP and deficits in long-term memory formation. ${ }^{50}$ Egr-1 expression is down-regulated markedly in visual deprivation, ${ }^{48}$ post-mortem brains of patients with schizophrenia $^{51}$ and exposure to social isolation stress. ${ }^{52}$ Similar to Arc expression, the Egr1 genes expression decreased in the IC and AC in rats with tinnitus following long-term administration of salicylate and returned to normality 14 days later, accompanied by disap- pearance of tinnitus. We hypothesize instability of long-lasting forms of homeostatic plasticity in tinnitus causes the decline of Egr-1 expression and this speculation requires further experimental support.

In conclusion, tinnitus induced with longterm salicylate administration was correlated with decreased expression of Arc and Egr-1 and increased expression of NR2B in the IC and AC. All returned to normal 14 days after treatment with salicylate ceased. These results
A

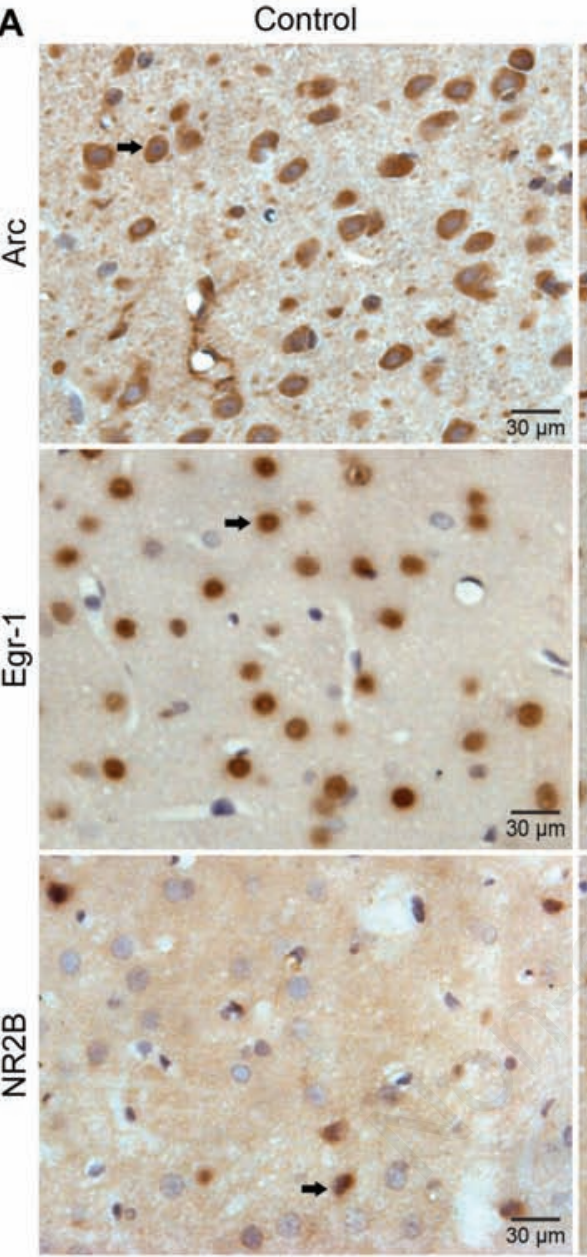

B

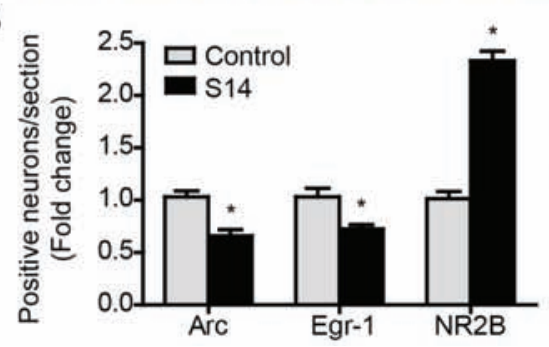

S14

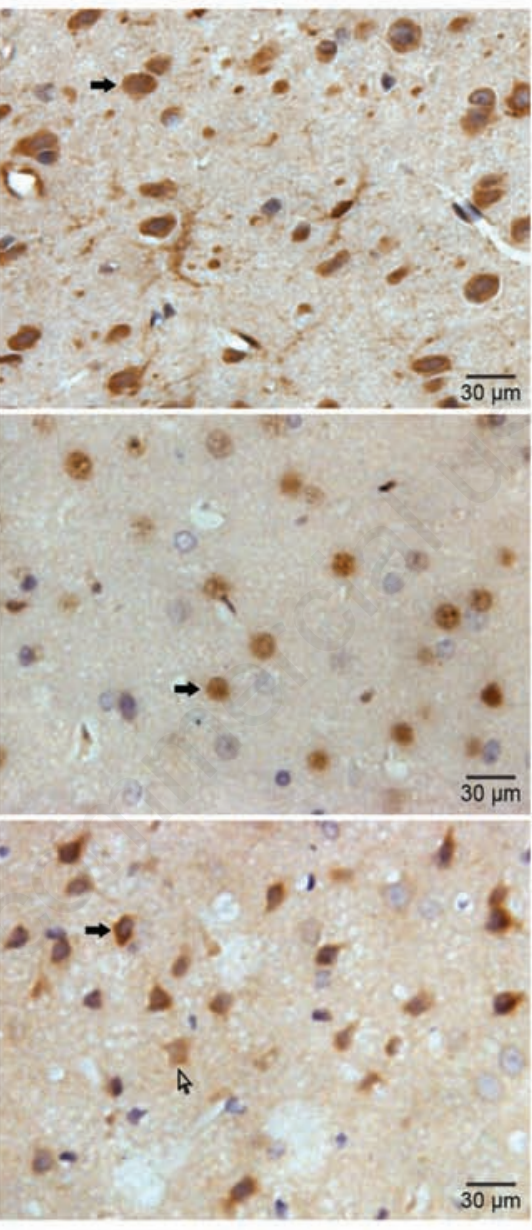

C

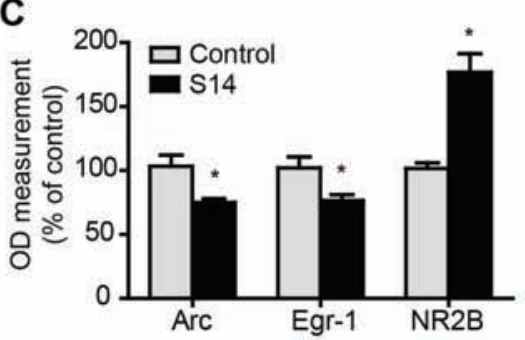

Figure 5. The expression of Arc, Egr-1 and NR2B was evaluated using immunohistochemistry staining (magnification $400 \times$ ) in the AC. A) Immunohistochemical staining of Arc, Egr-1 and NR2B in the AC. Black arrows indicate neurons and white arrows indicate glia. B) The number of Arc, Egr-1 and NR2B positively stained cells in the AC. C) Bars show percentage difference in OD compared to the control group (100\%). Values are given as mean $\pm S D, n=6 .{ }^{*} P<0.05 v$ s the control group. Scale bars: $30 \mu \mathrm{m}$.

showed long-term administration of salicylate induced tinnitus markedly but reversibly and caused neural changes of plasticity at the IC and AC level. Decreased expression of Arc and Egr-1 might serve as a marker of instability of long-lasting forms of synaptic plasticity.

\section{References}

1. Bartels H, Staal MJ, Albers FW. Tinnitus and neural plasticity of the brain. Otol Neurotol 2007;28:178-84.

2. Langguth B, Salvi R, Elgoyhen AB. Emerging pharmacotherapy of tinnitus. Expert Opin Emerg Drugs 2009;14: 687-702.

3. Jastreboff PJ, Brennan JF, Coleman JK, Sasaki CT. Phantom auditory sensation in rats: an animal model for tinnitus. Behav Neurosci 1988;102:811-22.

4. Paul AK, Lobarinas E, Simmons R, Wack D, Luisi JC, Spernyak J, et al. Metabolic imaging of rat brain during pharmacologicallyinduced tinnitus. Neuroimage 2009;44: $312-8$.

5. Yu N, Zhu ML, Johnson B, Liu YP, Jones RO, Zhao HB. Prestin up-regulation in chronic salicylate (aspirin) administration: an implication of functional dependence of prestin expression. Cell Mol Life Sci 2008; 65:2407-18.

6. Huang ZW, Luo Y, Wu Z, Tao Z, Jones RO, Zhao HB. Paradoxical enhancement of active cochlear mechanics in long-term administration of salicylate. J Neurophysiol 2005;93:2053-61.

7. Yang K, Huang ZW, Liu ZQ, Xiao BK, Peng JH. Long-term administration of salicylate enhances prestin expression in rat cochlea. Int J Audiol 2009;48:18-23.

8. Cazals Y, Huang ZW. Average spectrum of cochlear activity: a possible synchronized firing, its olivo-cochlear feedback and alterations under anesthesia. Hear Res 1996; 101:81-92.

9. Cazals Y, Horner KC, Huang ZW. Alterations in average spectrum of cochleoneural activity by long-term salicylate treatment in the guinea pig: a plausible index of tinnitus. $\mathrm{J}$ Neurophysiol 1998;80:2113-20.

10. Manabe Y, Saito T, Saito H. [Effects of lidocaine on salicylate-induced discharge of auditory neurons in the inferior colliculus of theguinea pig].[Article in Japanese]. Nihon Jibiinkoka Gakkai Kaiho 1998;101: 807-13.

11. Chen GD, Jastreboff PJ. Salicylate-induced abnormal activity in the inferior colliculus of rats. Hear Res 1995;82:158-78.

12. Eggermont JJ, Kenmochi M. Salicylate and quinine selectively increase spontaneous firing rates in secondary auditory cortex. 
Hear Res 1998;117:149-60.

13. Ochi K, Eggermont JJ. Effects of salicylate on neural activity in cat primary auditory cortex. Hear Res 1996;95: 63-76.

14. Wei L, Ding D, Sun W, Xu-Friedman MA, Salvi R. Effects of sodium salicylate on spontaneous and evoked spike rate in the dorsal cochlear nucleus. Hear Res 2010;267: 54-60.

15. Basta D, Goetze R, Ernst A. Effects of salicylate application on the spontaneous activity in brain slices of the mouse cochlear nucleus, medial geniculate body and primary auditory cortex. Hear Res 2008; 240:42-51.

16. Sagar SM, Sharp FR, Curran T. Expression of $\mathrm{c}$-fos protein in brain: metabolic mapping at the cellular level. Science 1988;240:1328-31.

17. Okuno H. Regulation and function of immediate-early genes in the brain: beyond neuronal activity markers. Neurosci Res 2011;69:175-86.

18. Cole AJ, Saffen DW, Baraban JM, Worley PF. Rapid increase of an immediate early gene messenger RNA in hippocampal neurons by synaptic NMDA receptor activation. Nature 1989;340:474-6.

19. Lobarinas E, Hayes SH, Allman BL. The gapstartle paradigm for tinnitus screening in animal models: limitations and optimization. Hear Res 2013;295:150-60.

20. Engineer ND, Riley JR, Seale JD, Vrana WA, Shetake JA, Sudanagunta SP, et al. Reversing pathological neural activity using targeted plasticity. Nature 2011;470:101-4.

21. Yang G, Lobarinas E, Zhang L, Turner J, Stolzberg D, Salvi R, et al. Salicylate induced tinnitus: behavioral measures and neural activity in auditory cortex of awake rats. Hear Res 2007;226:244-53.

22. Su YY, Luo B, Jin Y, Wu SH, Lobarinas E, Salvi RJ, et al. Altered neuronal intrinsic properties and reduced synaptic transmission of the rat's medial geniculate body in salicylate-induced tinnitus. PLoS One 2012;7:e46969.

23. Livak KJ, Schmittgen TD. Analysis of relative gene expression data using real-time quantitative PCR and the 2(-Delta Delta C(T)) Method. Methods 2001;25:402-8.

24. Guzowski JF, McNaughton BL, Barnes CA, Worley PF. Environment-specific expression of the immediate-early gene Arc in hippocampal neuronal ensembles. Nat Neurosci 1999;2:1120-4.

25. Maddox SA, Monsey MS, Schafe GE. Early growth response gene 1 (Egr-1) is required for new and reactivated fear memories in the lateral amygdala. Learn Mem 2011;18: 24-38.

26. Ramirez-Amaya V, Vazdarjanova A, Mikhael D, Rosi S, Worley PF, Barnes CA. Spatial exploration-induced Arc mRNA and protein expression: evidence for selective, networkspecific reactivation. J Neurosci 2005;25:
1761-8.

27. Tagawa Y, Kanold PO, Majdan M, Shatz CJ. Multiple periods of functional ocular dominance plasticity in mouse visual cortex. Nat Neurosci 2005;8:380-8.

28. Pinaud R. Experience-dependent immediate early gene expression in the adult central nervous system: evidence from enriched-environment studies. Int $\mathrm{J}$ Neurosci 2004;114:321-33.

29. Kaczmarek L, Chaudhuri A. Sensory regulation of immediate-early gene expression in mammalian visual cortex: implications for functional mapping and neural plasticity. Brain Res Brain Res Rev 1997;23:237-56.

30. Hwang JH, Chen JC, Chan YC. Effects of Cphycocyanin and Spirulina on salicylateinduced tinnitus, expression of NMDA receptor and inflammatory genes. PLoS One 2013;8:e58215.

31. Steward 0, Worley PF. Selective targeting of newly synthesized Arc mRNA to active synapses requires NMDA receptor activation. Neuron 2001;30:227-40.

32. Mokin M, Keifer J. Expression of the immediate-early gene-encoded protein Egr-1 (zif268) during in vitro classical conditioning. Learn Mem 2005;12:144-9.

33. Beckmann AM, Wilce PA. Egr transcription factors in the nervous system. Neurochem Int 1997;31:477-510; discussion 7-6.

34. Wenzel GI, Warnecke A, Stover T, Lenarz T. Effects of extracochlear gacyclidine perfusion on tinnitus in humans: a case series. Eur Arch Otorhinolaryngol 2010;267:691-9.

35. Rao VR, Pintchovski SA, Chin J, Peebles CL, Mitra S, Finkbeiner S. AMPA receptors regulate transcription of the plasticity-related immediate-early gene Arc. Nat Neurosci 2006;9:887-95.

36. Shepherd JD, Rumbaugh G, Wu J, Chowdhury S, Plath N, Kuhl D, et al. Arc/Arg3.1 mediates homeostatic synaptic scaling of AMPA receptors. Neuron 2006;52:475-84.

37. Goel A, Lee HK. Persistence of experienceinduced homeostatic synaptic plasticity through adulthood in superficial layers of mouse visual cortex. J Neurosci 2007;27: 6692-700.

38. Okuno H, Akashi K, Ishii Y, Yagishita-Kyo N, Suzuki K, Nonaka M, et al. Inverse synaptic tagging of inactive synapses via dynamic interaction of Arc/Arg3.1 with CaMKIIbeta. Cell 2012;149:886-98.

39. Moser EI, Krobert KA, Moser MB, Morris RG. Impaired spatial learning after saturation of long-term potentiation. Science 1998;281: 2038-42.

40. Turrigiano GG, Leslie KR, Desai NS, Rutherford LC, Nelson SB. Activity-dependent scaling of quantal amplitude in neocortical neurons. Nature 1998;391:892-6.

41. Ruttiger L, Singer W, Panford-Walsh R,
Matsumoto M, Lee SC, Zuccotti A, et al. The reduced cochlear output and the failure to adapt the central auditory response causes tinnitus in noise exposed rats. PLoS One 2013;8:e57247.

42. Peebles CL, Yoo J, Thwin MT, Palop JJ, Noebels JL, Finkbeiner S. Arc regulates spine morphology and maintains network stability in vivo. Proc Natl Acad Sci U S A 2010;107:18173-8.

43. Jastreboff PJ, Sasaki CT. An animal model of tinnitus: a decade of development. Am J Otol 1994;15:19-27.

44. Singer W, Zuccotti A, Jaumann M, Lee SC, Panford-Walsh R, Xiong $\mathrm{H}$, et al. Noiseinduced inner hair cell ribbon loss disturbs central arc mobilization: a novel molecular paradigm for understanding tinnitus. Mol Neurobiol 2013;47:261-79.45.

45. Alberini CM. Transcription factors in longterm memory and synaptic plasticity. Physiol Rev 2009;89:121-45.

46. Waltereit R, Dammermann B, Wulff P, Scafidi J, Staubli U, Kauselmann G, et al. Arg3.1/Arc mRNA induction by $\mathrm{Ca} 2+$ and cAMP requires protein kinase $\mathrm{A}$ and mitogen-activated protein kinase/extracellular regulated kinase activation. J Neurosci 2001;21:5484-93.

47. Davis S, Vanhoutte P, Pages C, Caboche J, Laroche $\mathrm{S}$. The MAPK/ERK cascade targets both Elk-1 and cAMP response elementbinding protein to control long-term potentiation-dependent gene expression in the dentate gyrus in vivo. J Neurosci 2000;20: 4563-72.

48. Mataga N, Fujishima S, Condie BG, Hensch TK. Experience-dependent plasticity of mouse visual cortex in the absence of the neuronal activity-dependent marker egr1/zif268. J Neurosci 2001;21:9724-32.

49. Davis S, Bozon B, Laroche S. How necessary is the activation of the immediate early gene zif268 in synaptic plasticity and learning? Behav Brain Res 2003;142: 17-30.

50. Jones MW, Errington ML, French PJ, Fine A, Bliss TV, Garel S, et al. A requirement for the immediate early gene Zif268 in the expression of late LTP and long-term memories. Nat Neurosci 2001;4:289-96.

51. Yamada K, Gerber DJ, Iwayama Y, Ohnishi T, Ohba H, Toyota T, et al. Genetic analysis of the calcineurin pathway identifies members of the EGR gene family, specifically EGR3, as potential susceptibility candidates in schizophrenia. Proc Natl Acad Sci USA 2007;104:2815-20.

52. Matsumoto K, Ono K, Ouchi H, Tsushima R, Murakami Y. Social isolation stress downregulates cortical early growth response 1 (Egr-1) expression in mice. Neurosci Res 2012;73:257-62. 